Historic, Archive Document

Do not assume content reflects current scientific knowledge, policies, or practices. 



\title{
1926 Price List
}

"1

\section{ROOT \& ST. GEORGE}

111

CHARLOTTE, VERMONT

\section{Collectors of Hardy Plants, Shrubs, Evergreens, Etc.}

TERMS, CONDITION OF SALE, ETC.

\begin{abstract}
All bills are due and payable thirty days from date of invoice, unless otherwise arranged. We do not warrant any trees, shrubs or plants sold by us except as to genuineness. The purchaser assumes all risk of transportation. Boxing and packing are charged at cost. Not less than 25 or 250 unless otherwise arranged for, will be furnished at 100 and 1,000 rates, respectively. All claims for errors must be reported immediately upon receipt of goods and will be rectified promptly.
\end{abstract}

\section{HARDY PLANTS AND ORCHIDS}

Name

Per Per

Name

Per Per

$100 \quad 1,000$

Actaea Alba

$\$ 5.00 \$ 40.00$

Actaea Rubra

5.00

Aralia Racemosa

10.00

$3.00 \quad 25.00$

$3.00 \quad 25.00$

Anemone Nemorosa

$3.00 \quad 20.00$

Anemone Canadensis

4.00

Anemonella Thalictroides

Aposynum Androsaemifolium

$4.00 \quad 30.00$

Aquilegia Canadensis

$3.00 \quad 25.00$

Asarum Canadense

$3.00 \quad 20.00$

Aster Novae Angliac

$4.00 \quad 35.00$

Aster Novae Angliac Var. Roseus

Arisaema Triphillum

3.00

25.00

Campanula Rotundifolia

$3.00 \quad 25.00$

Caltha Palustris

3.00

20.00

Chelone Glabra

$3.00 \quad 20.00$

Chimaphila Umbellata

3.00

20.00

Claytonia Caroliniana

$3.00 \quad 20.00$

Clintonia Borealis

Convallaria Majalis

$3.00 \quad 25.00$

$5.00 \quad 40.00$

Coptis Trifolia

3.00

20.00

Caulophyllum Thalictroides

$6.00 \quad 50.00$

100

1,000

Cornus Canadensis

Corrallorrhiza Maculata

Dentaria Diphylla

Dicentra Cucullaria

Dicentra Canadensis

Erythronium Americanum

Eupatorium Urticaefolium

Eupatorium Perfoliatum

Eupatorium Purpureum

Gaultheria Procumbens

Gentiana Andrewsii

Geranium Robertianum

Goodyera Pubescens

Goodyera Repens

Hepatica Acutiloba

Hepatica Triloba

Houstonia Caerulea

Iris Versicolor

Lilium Canadense

Lilium Philadelphicum

Lobelia Cardinalis

Lupinus Perennis

Mentha Piperita
$\$ 3.00 \$ 20.00$

8.00

$3.00 \quad 20.00$

$5.00 \quad 40.00$

$4.00 \quad 30.00$

$3.00 \quad 20.00$

$4.00 \quad 30.00$

$4.00 \quad 30.00$

$4.00 \quad 30.00$

$3.00 \quad 20.00$

$4.00 \quad 30.00$

$3.00 \quad 20.00$

$5.00 \quad 40.00$

$5.00 \quad 40.00$

$3.00 \quad 20.00$

$3.00 \quad 20.00$

$3.00 \quad 20.00$

$5.00 \quad 40.00$

$6.00 \quad 50.00$

$5.00 \quad 40.00$

$5.00 \quad 40.00$

$5.00 \quad 40.00$

5.00 


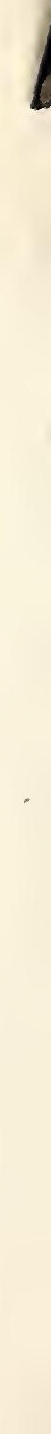




\begin{tabular}{|c|c|c|c|c|c|}
\hline Name & $\begin{array}{l}\text { Per } \\
100\end{array}$ & $\begin{array}{l}P e r \\
1,000\end{array}$ & Name & $\begin{array}{l}\text { Per } \\
100\end{array}$ & $\begin{array}{l}\text { Per } \\
1,000\end{array}$ \\
\hline Mentha Spicata & $\$ 5.00$ & & Aspidium Spinuloeum Var. & & \\
\hline Mitchella Repens & 3.00 & & Intermedium & $\$ 3.00$ & $\$ 25.00$ \\
\hline Mitella Diphylla & 4.00 & $\$ 30.00$ & Aspidium Thelypteris & 3.00 & 25.00 \\
\hline Oakesia Sessilifolia & 3.00 & 20.00 & Asplenium Augustifolium & 8.00 & \\
\hline Orchis Spectabilis & 5.00 & & Asplenium Ebeneum & 5.00 & 40.00 \\
\hline Polygala Paucifolia & 3.00 & 25.00 & Asplenium Filix-foemina & 5.00 & 40.00 \\
\hline Polygala Senega & 4.00 & 30.00 & Asplenium Trichomanes & 5.00 & 40.00 \\
\hline Polygonatum Biflorum & 5.00 & & Asplenium Acrostichoides & 5.00 & 40.00 \\
\hline Pyrola Elliptica & 4.00 & 30.00 & Camptosorus Rizaphyllus & 5.00 & \\
\hline Sanguinaria Canadensis & 3.00 & 20.00 & Cystopteris Bulbifera & 4.00 & 30.00 \\
\hline Saxifraga Virginiensis & 3.00 & 20.00 & Cystopteris Fragilis & 4.00 & 30.00 \\
\hline Sedum Acre & 4.00 & 30.00 & Dicksonia Punctilobula & 3.00 & 25.00 \\
\hline Sedum Triphyllum & 4.00 & 30.00 & Onoclea Sensibilis & 3.00 & 25.00 \\
\hline Sarraecenia Purpurea & 4.00 & 30.00 & Onoclea Struthßopteris & 3.00 & 25.00 \\
\hline Smilacina Racemosa & 3.00 & 20.00 & Phegopteris Dryopteris & 3.00 & \\
\hline Smilacina Bifolia & 3.00 & 20.00 & Phegopteris Polypodioides & 3.00 & \\
\hline Solidago in Variety & 4.00 & 30.00 & Polypodium Vulgare & 3.00 & 25.00 \\
\hline Spiranthes Cernua & 4.00 & 30.00 & Woodsia Ilvensis & 4.00 & 30.00 \\
\hline Spiranthes Gracilis & 5.00 & & Woodsia Obtusa & 5.00 & \\
\hline Thalictrum Dioicum & 3.00 & 20.00 & Osmunda Cinnamomea & 5.00 & 40.00 \\
\hline Thalictrum Polygamum & 4.00 & 30.00 & Osmunda Claytoniana & 5.00 & 40.00 \\
\hline Trillium Erectum & 3.00 & 20.00 & Osmunda Regalis & 5.00 & 40.00 \\
\hline Trillium Grandiflorum & 2.00 & 15.00 & Botrychium Virginianum & 5.00 & \\
\hline Trillium Undulatum & 5.00 & & HARDY EVERGREENS & & \\
\hline Trientalis Americana & 3.00 & 20.00 & Juniperus Communis & & \\
\hline Tiarella Cordifolia & 3.00 & 25.00 & $4-8$ in. & 4.00 & 30.00 \\
\hline Uvularia Grandiflora & 3.00 & 20.00 & $8-12$ in. & 7.00 & 60.00 \\
\hline Viola Canadensis & 4.00 & 30.00 & Juniperus Virginiana & & \\
\hline Viola Pubescens & 4.00 & 30.00 & $4-8$ in. & 3.00 & 25.00 \\
\hline Viola Cucullata & 4.00 & 30.00 & $8-12$ in. & 5.00 & 40.00 \\
\hline $\begin{array}{l}\text { Viola Rotundifolia } \\
\text { HARDY ORCHII }\end{array}$ & 4.00 & 30.00 & Picea Canadensis & & \\
\hline FLOWERING BUDS & & & $4-8$ in. & 4.00 & 30.00 \\
\hline Cypripedium Acaule & 5.00 & 40.00 & $8-12$ in. & 5.00 & 40.00 \\
\hline Cypripedium Parviflorum & 7.00 & 60.00 & Taxus Canadensis & & \\
\hline Cypripedium Var. Pubescens & 7.00 & 60.00 & 6-12 in. & 4.00 & 30.00 \\
\hline Cypripedium Hirsutum & 7.00 & 60.00 & Tsuga Canadensis & & \\
\hline HARDY FERNS & & & $2-5$ in. & 2.00 & 15.00 \\
\hline Adiantum Pedatum & 3.00 & 20.00 & $5-8$ in. & 3.00 & 25.00 \\
\hline Aspidium Acrostichoides & 3.00 & 25.00 & $8-12$ in. & 4.00 & 35.00 \\
\hline Aspidium Cristatum & 5.00 & & Thuja Occidentalis & & \\
\hline Aspidium Var. Clintonianum & 5.00 & & 3-5 in. & & 5.00 \\
\hline Aspidium Marginale & 3.00 & 25.00 & $5-8$ in. & & 10.00 \\
\hline Aspidium Noveboracense & 4.00 & 30.00 & $8-12$ in. & 3.00 & 25.00 \\
\hline
\end{tabular}


Name Per Per

$100 \quad 1,000$

HARDY SHRUBS

Amelanchier Canadensis

$1-3 \mathrm{ft}$.

$\$ 4.00$

Acer Saccharum

$1-2 \mathrm{ft}$.

$3.00 \$ 20.00$

Betula Alba Var. Papyrifera

$2-3 \mathrm{ft}$.

$4.00 \quad 35.00$

Betula Populifolia

$2-3 \mathrm{ft}$.

$4.00 \quad 35.00$

Cornus Alternifolia

$1-3 \mathrm{ft}$.

4.00

Cornus Circinata

$1-3 \mathrm{ft}$.

4.00

Cornus Amomum

$1-3 \mathrm{ft}$.

$4.00 \quad 30.00$

Cornus Stolonifera

$1-3 \mathrm{ft}$.

$4.00 \quad 30.00$

Daphne Mezereum

$4-8$ in.

$10-18$ in.

$10.00 \quad 80.00$

$20.00 \quad 175.00$

Ilex Verticillata

$1-3 \mathrm{ft}$.

$4.00 \quad 30.00$

Myrica Asplenifolia

$4.00 \quad 30.00$

Pyrus Americana

$1-3 \mathrm{ft}$.

10.00

Rhus Copallina

$1-2 \mathrm{ft}$.

$5.00 \quad 40.00$

Rhus Typhina

$1-3 \mathrm{ft}$.

$4.00 \quad 30.00$

Rubus Odoratus

$1-3 \mathrm{ft}$.

$3.00 \quad 25.00$

Rhodora Canadensis flowering bush

25.00

Sambucus Canadensis $1-3 \mathrm{ft}$.

$3.00 \quad 20.00$

Sambucus Racemosa $1-3 \mathrm{ft}$.

$10.00 \quad 80.00$

Viburnum Accrifolium $1-3 \mathrm{ft}$.

$5.00 \quad 40.00$

Viburnum Cassinoides 8-18 in.

$3.00 \quad 25.00$

Clumps

$1-3 \mathrm{ft}$.

$6.00 \quad 50.00$ 


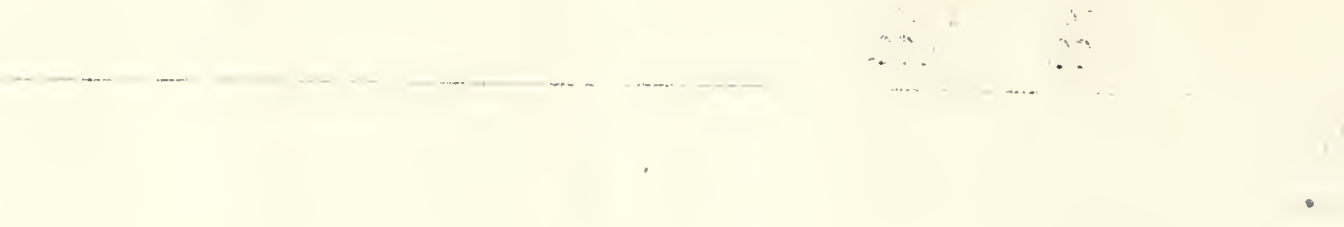

